\title{
Numerical simulation of porous media combustion for high temperature heat exchanger
}

\author{
Panu Iamsakulpanich ${ }^{1}$, Kittipass Wasinarom ${ }^{2}$, Thanathon Sesuk ${ }^{3}$, Jarruwat Charoensuk ${ }^{2}$, Katsunori Hanamura ${ }^{4}$, \\ Preecha Karin $^{1}$, and Visarn Lilavivat ${ }^{3 *}$ \\ ${ }^{1}$ Automotive engineering, International College, King Mongkut's Institute of Technology Ladkrabang, Bangkok, Thailand \\ ${ }^{2}$ Department of Mechanical Engineering, Faculty of Engineering, King Mongkut's Institute of Technology Ladkrabang, \\ Bangkok, Thailand \\ ${ }^{3}$ Materials for energy research unit, National metal and materials technology center (MTEC), Patumthani, Thailand \\ ${ }^{4}$ Tokyo Institute of Technology, Tokyo, Japan
}

\begin{abstract}
The purpose of this work is developing the numerical 1D model of porous media combustion for investigating porous media burner systems. The software is used to solve energy, mass transfer and chemical reaction equation of the combustion. The operating condition and property parameters, which mainly affect the functions and quality of the industrial burner design, such as the inlet velocity of the reactants, the equivalence ratio, the extinction coefficient and the thermal conductivity of porous media, will be investigated and validated with experimental data. For developing the procedure of experiment, three diameter sizes of porous media materials $(5 \mathrm{~mm}, 10$ $\mathrm{mm}$, and $15 \mathrm{~mm}$.) were used. As a result, the developed model will be used as a tool to explore temperature distribution of heat exchange to improve thermal performance and overall efficiency system. Moreover, this knowledge can be applied to design porous media burner systems for uniform temperature distribution operation.
\end{abstract}

\section{Introduction}

In the present, environmental concerns and global warming problems have an impact on our environment. Although, the sustainable energy such as biomass, hydropower, geothermal, solar, wind and marine energies has been progressively used, it still cannot supply enough for the total world energy. And the nuclear energy has a problem about safety. Furthermore, the world considers reducing the consumption of coal and petroleum to reduce greenhouse gases. Under these conditions, improve the efficiency of in the present combustion has important.

Currently, the heat exchanger is widely used in commercial industry such as power stations, chemical plants and petrochemical plants etc. Operating of the heat exchanger in commercial industry is transfer heat from combustion to use. high temperature heat exchanger is an improved efficiency of the heat exchanger in high temperature operating condition but they have problems about meltdown of material, how to design heat transfer in heat exchanger etc.

Accordingly, porous media materials in the combustion of heat exchanger have attracted attention for improving heat exchanger efficiency in this work because of their advantages such as higher burning rates than free flame, extended lean flammability limits, low emissions of pollutants, high radiant output, and increased power dynamic range.

This paper presents the development of the combustion modeling in porous media. The developing code is based on "One-dimensional CFD combustion modeling in porous media" written by Wasinarom[1]. The model is governed by conservation of mass, fuel species, energy in fluid pore space, energy in a solid porous matrix. According to the 2 phases of Heat transfer between solid and fluid phase, local thermal nonequilibrium was been modeling via energy source term of the solid and fluid energy equation. Arrhenius law is used to calculate fuel consumption and heat transfer from oxidation. The code is developed in one dimension due to prevent fluid dynamics complication at this stage. And, velocity field was merely calculated by ideal gas law as the calorific equation of state with $1 \mathrm{D}$ models. One dimensional code is recommended at the early stage of the complex porous media with combustion development.

\section{Methodology}

\subsection{Porous media combustion modeling (PMC modeling)}

The porous media combustion modeling trends up to 1994[2] are mainly described 1D models only. Takeno, Echigo, and their coworkers[3] are the pioneers in PMC modeling to study the effects of mass flow rate and heat transfer coefficient on flame characteristics in excess enthalpy flames and suggested inserting a porous, highly conductive solid into the flame to conduct heat from the solid to the reactants. They found that increasing the mass flow rate above the laminar burning rate increased the heat

\footnotetext{
* Corresponding author: visarn.lil $@$ mtec.or.th
} 
release rate and the reaction zone became more concentrated.

Generally, all 1D models assume 1D flow conditions and no radial heat losses[4]. These two assumptions may become inaccurate if the porous media combustor is a commercial burner prototype with complex geometry. In such a situation 1D relations will no longer be valid and multidimensional models are imperative. It is, therefore, necessary to predict 3D combustion and emissions in complex geometrical burner configurations to help the design of commercial inert porous burners[5].

Mohamad et al.[6,7] modeled a PM burner with embedded coolant tubes. The 2D continuity, momentum, energy and fuel mass fraction equations were solved and the combustion was described as a one-step reaction. A numerical code has been developed by Bidi et al.[8] to evaluate the effects of different parameters of combustion in porous media. The Navier-Stokes, the solid and gas energy and the chemical species transport equations were solved using a multi-step reduced kinetic mechanism. The discrete ordinates method was used to solve the radiative transfer equation and a finite volume method (FVM) based on SIMPLE method was applied to discretize the conservation equations. Moraga et al.[9] who studied the convective heat transfer within a cylindrical inert porous media combustor have used a $2 \mathrm{D}$, two temperature mathematical model, based on fluid mechanics, energy and chemical species governing equations The FVM was used to solve the discrete model for methane combustion with air.

Hayashi and coworkers[10] had introduced the 3D modeling of a two-layer burner. The first layer was a perforated plate made of an insulating material $\left(\mathrm{Al}_{2} \mathrm{O}_{3}\right)$ with the purpose of avoiding flash-back, while the second layer, a thin plate made of $\mathrm{SiC}$ foam to act as the reaction layer. They claimed that the proposed 3D model could facilitate a detailed study of the flow at the interface of the two solid layers, which is not possible utilizing one- or two-dimensional models, owing to the complex flow structure originated by the $3 \mathrm{D}$ jets from the perforated plate into the $\mathrm{SiC}$ foam.

For most of the cases, a 1D model with single-step reaction kinetics could yield the results with reasonable accuracy. In fact, the realistic prediction of pollutants formation necessitated detailed reaction kinetics to be incorporated in the model. And in $3 \mathrm{D}$ simulation, the inclusion of detailed reaction chemistry is still lacking, or $3 \mathrm{D}$ simulation is too small[11].

\subsection{Porous media burner}

Porous media combustion has three modes of heat transfer conduction, convection, and radiation. Advantages of Porous media burner[12] is a better homogenization of temperature across the porous media and the significant amount of radiation helps to preheat the incoming air-fuel mixture at upstream. The technique of premixed combustion within porous media has been studied and applied to steady combustion with great success. The porous media combustion has proved to be one of the applicable options to solve the problems to a remarkable extent in both technical and economic perspectives. This technique has[12] been used for both gaseous and liquid fuels in steady or unsteady combustion. Flame stability in porous media with lean and rich mixtures, a significant reduction in pollutants and increasing combustion efficiency, was proven.

The material of porous media burner[13], aluminum oxide $\left(\mathrm{Al}_{2} \mathrm{O}_{3}\right)$, silicon carbide $(\mathrm{SiC})$, and zirconium dioxide $\left(\mathrm{ZrO}_{2}\right)$ proposed as suitable materials for application. Aluminum oxide and zirconium dioxide were recognized as high temperature resistant materials. SiC shows good thermal shock resistance, mechanical strength, and conductive heat transport. Silicon carbide also has a high melting point $(3260 \mathrm{~K})$, against cyclic thermal stress and strength retention at the peak regenerator temperature $(1673 \mathrm{~K})$, and excellent oxidation resistance. Metallic materials were found less suitable for porous media because of their inadequate thermal stability and high thermal inertia.

A comparison of the data for materials relevant for use in porous media burners is shown in Table 1.

Table 1 Most important material data for $\mathrm{Al}_{2} \mathrm{O}_{3}, \mathrm{SiC}$ and $\mathrm{ZrO}_{2}$ [14]

\begin{tabular}{|l|c|c|c|c|}
\hline \multicolumn{1}{|c|}{ Property } & $\begin{array}{c}\text { Uni } \\
\mathbf{t}\end{array}$ & $\mathbf{A l}_{2} \mathbf{O}_{3}$ & $\mathbf{S i C}$ & $\mathbf{Z r O}_{2}$ \\
\hline $\begin{array}{l}\text { Maximum use } \\
\text { temperature in air }\end{array}$ & ${ }^{\circ} \mathrm{C}$ & 1900 & 1650 & 1800 \\
\hline $\begin{array}{l}\text { Thermal expansion } \\
\text { coefficient } \\
\left(20-1000^{\circ} \mathrm{C}\right)\end{array}$ & $\begin{array}{c}10^{-6} \\
K^{-1}\end{array}$ & 8 & $4-5$ & $10-13$ \\
\hline $\begin{array}{l}\text { Thermal conductivity } \\
\text { at } 20^{\circ} \mathrm{C}\end{array}$ & $\begin{array}{c}W \\
m^{-1} \\
K^{-1}\end{array}$ & $20-30$ & $80-$ & $2-5$ \\
& $\begin{array}{l}\text { Thermal conductivity } \\
\text { at } 1000{ }^{\circ} \mathrm{C}\end{array}$ & $5-6$ & $20-$ & $2-4$ \\
$m^{-1}$ & $K^{-1}$ & & 50 & \\
\hline $\begin{array}{l}\text { Specific thermal } \\
\text { capacity }\end{array}$ & $J g^{-1}$ & $0.9-1$ & $0.7-$ & $0.5-$ \\
$K^{-1}$ & $K$ & 100 & 230 & 230 \\
\hline $\begin{array}{l}\text { Thermal stress } \\
\text { resistance parameter, } \\
\text { hard shock, R }(\sigma / \mathrm{E} \alpha)\end{array}$ & & & & \\
\hline $\begin{array}{l}\text { Thermal stress } \\
\text { resistance parameter, } \\
\text { mild thermal shock, } \\
\text { R' (R } \lambda \text { ) }\end{array}$ & $10^{-3}$ & 3 & 23 & 1 \\
\hline
\end{tabular}

\section{Experiment section}

\subsection{Experiment setup}

Fig. 1 shows the schematic of the experimental setup. This system is composed of methane gas and air supply systems, a mixing chamber, four sizes of porous media, and a measurement system. Methane gas and air were controlled by MFC and then mixed in the mixing chamber. The methane/air mixture flowed through flashback protection and after that combustion in a quartz tube $75 \mathrm{~mm}$. in diameter and $170 \mathrm{~mm}$. long. 


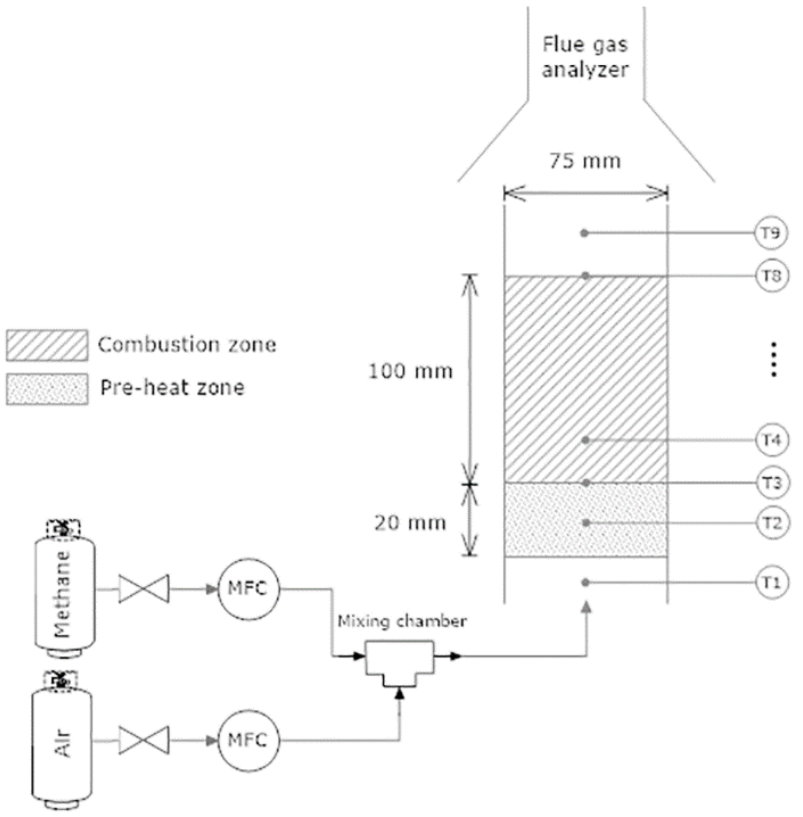

Fig. 1 Schematic of the experimental setup.

In the burner, a quartz tube was separated into two layers of porous media. The first layer was a preheat zone that was $20 \mathrm{~mm}$. long and filled with $\mathrm{Al}_{2} \mathrm{O}_{3}$ pellets diameter size $3.0 \mathrm{~mm}$. The second layer was the flame region that was $100 \mathrm{~mm}$. long and filled with $\mathrm{Al} 2 \mathrm{O} 3$ pellets diameter size $5.0 \mathrm{~mm}$. in the $1^{\text {st }}$ experiment, 10 $\mathrm{mm}$. in the $2^{\text {nd }}$ experiment, and $15 \mathrm{~mm}$. in the $3^{\text {rd }}$ experiment.

Thermocouples were set up as No.1 in the premixing zone and No.2 is in center of flashback protection zone. The No.3 is on the border between flashback protection zone and combustion zone. Thermocouples No.4-8 begin to start apart from No.3 in every $20 \mathrm{~mm}$ until No. 9 set depart of the combustion zone. The recorded data is temperature data and emission of the combustion exhausts such as $\mathrm{CO}, \mathrm{O}_{2}, \mathrm{NO}, \mathrm{C}_{\mathrm{x}} \mathrm{H}_{\mathrm{y}}$ and $\mathrm{NO}_{2}$ at the burner outlet.

The air flow rate at inlet burner of methane/air mixture is kept constant between $0.09-0.15 \mathrm{~m} / \mathrm{s}$. And, equivalent ratio is developed between 0.5-1.0 that was suggested by Marker J. et al.[15].

\subsection{Modeling equations}

For simplify the problem, Model was followed under the below assumptions. media.

1. The porous media was setup as gray homogeneous

2. The wall is no slip, adiabatic, and radiative gray.

3. In high temperature, Potential catalytic effects of the solid are ignored.

4. Gas radiation and Body forces is neglected.

5. Reactants and Products setup as incompressible ideal gases. form.

A set of differential equations is in the following

Continuity equation,

$$
\frac{\partial \varepsilon \rho_{g}}{\partial t}+\nabla \cdot\left(\varepsilon \rho_{g} u\right)=0
$$

Where

$\rho_{g}$ is the density of methane/air mixture

$\varepsilon$ is the porosity, $u$ is the velocity

Momentum equation,

$\varepsilon \rho_{g}\left(\frac{\partial u}{\partial t}+u \cdot \nabla u\right)=-\varepsilon \nabla \mathrm{p}+\mu \nabla^{2} u+\frac{1}{3} \mu \nabla(\nabla \cdot u)$

Where

$\mu$ is the viscosity of gas.

Species conservation equation,

$\frac{\partial \varepsilon \rho_{g} Y_{i}}{\partial t}+\nabla \cdot\left(\varepsilon \rho_{g} Y_{i}\right)=-\nabla \cdot\left(\varepsilon \rho_{g} Y_{i} V_{i}\right)+\varepsilon d$.

Where

$Y_{i}$ is the molar faction of substance

$V_{i}$ is the diffuse velocity of substance

d is the reaction rate of substance

$W_{i}$ is the molar mass of substance

Energy equation,

For gas

$$
\begin{aligned}
& \frac{\partial\left(\varepsilon \rho_{g} C_{g} T_{g}\right)}{\partial t}+\nabla \cdot\left(\varepsilon u\left(\rho_{g} C_{g} T_{g}+p\right)\right) \\
& \quad=h_{v}\left(T_{s}-T_{g}\right)+\nabla \cdot\left(\varepsilon k_{g}\right) \nabla T_{g}+\varepsilon d
\end{aligned}
$$

For solid

$\frac{\partial\left[(1-\varepsilon) \rho_{s} C_{s} T_{s}\right]}{\partial t}=\nabla \cdot\left(k_{s} \nabla T_{g}\right)+h_{v}\left(T_{s}-T_{g}\right)$

Where

$d$ is the heat from the chemical reaction

$h_{v}$ is the convection of heat transfer coefficient

$k_{s}$ is the heat transfer coefficient for solids

The inlet velocity is defined by

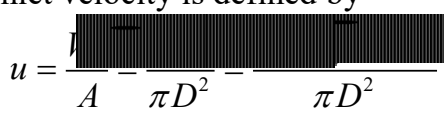

Where

is the gas flow rate of methane/air mixture

$A$ is the sectional area of the flow path

$D$ is the diameter of the flow path

I. III in the gas flow rates of methane

INWWin the gas flow rates of air

The porosity is defined as

$$
\operatorname{Porosity}(\varepsilon)=\frac{V_{\text {cavity }}}{V_{\text {total }}} \times 100
$$

Where

$\varepsilon$ is the ratio of the void volume within a porous media to the total bulk volume of the porous media

$V_{\text {Cavity }}$ is a volume of cavity of porous media pack bed

$V_{\text {total }}$ is a volume of cylindrical quartz tube 


\section{Results}

In this study, the combustion position occurs at the rapidly changed temperature of the fluid. And the location of the combustion is depended upon the porosity. The porosity. At the porosity of 0.30 or $5 \mathrm{~mm}$. of diameter size of porous media has the combustion position at approximately $1.9 \mathrm{~cm}$., and porosity of 0.35 (diameter size $10 \mathrm{~mm}$ ) has the combustion position at $2.2 \mathrm{~cm}$., and porosity of 0.40 (diameter size $15 \mathrm{~mm}$.) has the combustion position at $2.5 \mathrm{~cm}$., respectively

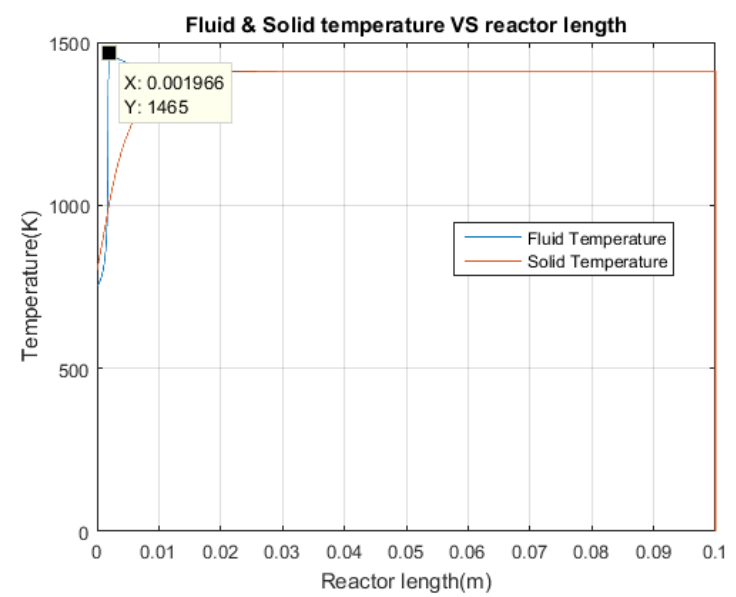

(a)

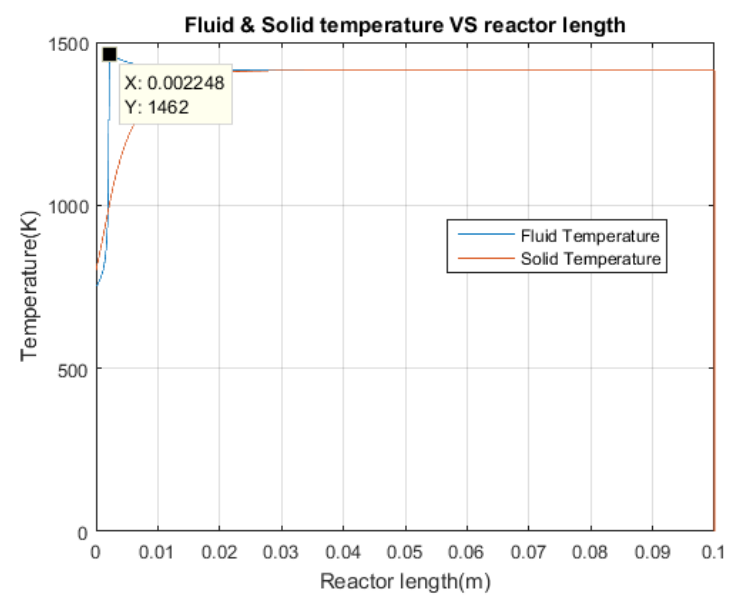

(b)

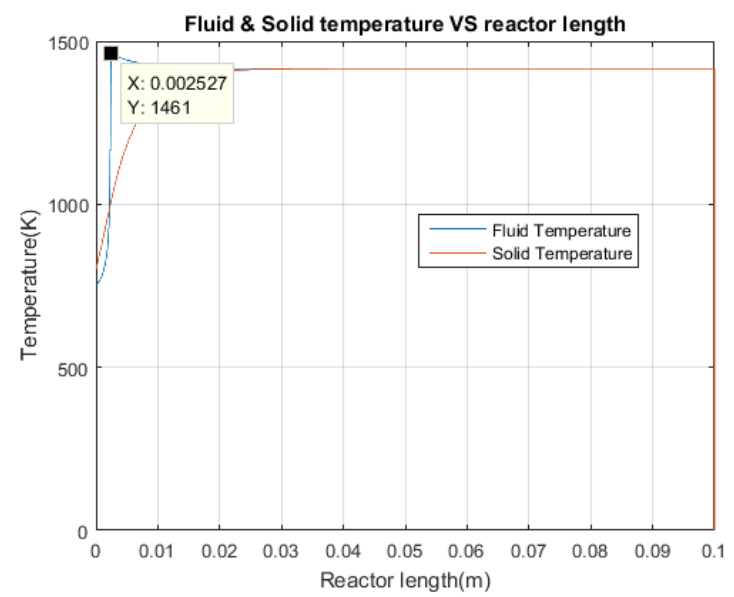

(c)

Fig. 2 Temperature distribution along the axial distance of modeling at porosity (a) 0.3 , (b) 0.35 , and (c) 0.4

\section{Conclusions}

The comparison three diameter size of porous media model results, the porosity of porous media affects the position of combustion that occurs in the model. The lower value of porosity shows the faster combustion will occurred.

This work was supported by National metal and materials technology center (MTEC).

\section{References}

1. K. Wasinarom, K. Onthong, D. Boonchauy, J. Charoensuk, TSME ICOME DECEMBER2016., (2016).

2. M. A. Mujeebu, M. Z. Abdullah, A. A. Mohamad, and M. Z. A. Bakar, Prog. Energy Combust. Sci., 36, 627-650 (2010).

3. T. Takeno and K. Sato, Combust. Sci. Technol. (1979).

4. A. Loukou et al., Int. J. Hydrogen Energy, 42, 652663 (2017)

5. I. MALICO, X. Y. ZHOU, J. C. F. PEREIRA, and J. C. F. Pereira, Combust. Sci. Technol., 152, 57-79 (2000)

6. A. A. MOHAMAD et al., Combust. Sci. Technol., 96, 387-407 (1994)

7. A. A. Mohamad, S. Ramadhyani, and R. Viskanta, Int. J. Heat Mass Transf. 37, 1181-1191 (1994).

8. M. Bidi, M. Saffar Avval, and M. Heyrani Nobari, in Eurotherm Seminar $n^{\circ} 81$ Reactive Heat Transfer in Porous Media, (2007).

9. N. O. Moraga, C. E. Rosas, V. I. Bubnovich, and N. A. Solari, Int. J. Heat Mass Transf. (2008).

10. T. C. Hayashi, I. Malico, and J. C. F. Pereira, in Computers and Structures, (2004).

11. V. Vandadi and C. Park, Energy (2016).

12. Z. Masoud and M. Arash, Numerical Simulation From Theory to Industry, chapter 22, Intech (2012).

13. P. Chitmongkol, thesis, RMUTT (2014).

14. F. Avdic, Thesis, Universität Erlangen-Nürnberg, (2004)

15. T. D. B. Mark J. Khinkis, Hamid A. Abbasi, PATENT, US5476375A (1994) 